\title{
COMPLETELY REDUCIBLE NEAR-RINGS
}

\author{
by A. OSWALD \\ (Received 11th March 1975)
}

To establish our notation $N$ will always denote a (left) near-ring without any type of multiplicative identity (unless the contrary is stated) satisfying $0 n=0$ for each $n \in N$ where 0 is the additive identity of $N$. A group $M$, written additively, which admits $N$ as a set of right multipliers is a (right) $N$-module if $a \in M, n_{1}, \quad n_{2} \in N$ implies $a\left(n_{1}+n_{2}\right)=a n_{1}+a n_{2}$ and $a\left(n_{1} n_{2}\right)=\left(a n_{1}\right) n_{2}$. When $N$ has a two-sided identity, 1 , we suppose that $a 1=a$ for each $a \in M$. A subgroup $X$ of $M$ is an $N$-subgroup of $M$ if it is an $N$-module; $X$ is a submodule of $M$ if it is a normal subgroup of $M$ and $a \in M, x \in X, n \in N$ implies $(a+x) n-a n \in X$. We denote by $S L(M)$ the set of $N$-subgroups and by $L(M)$ the set of submodules of $M$. Since $N$ may be regarded as an $N$-module we can talk about $N$-subgroups and submodules of $N$ although we usually call the submodules of $N$ right ideals of $N$. Other definitions can be found in (6).

An $N$-subgroup $A$ of $M$ is semicomplemented if there exists $B \in L(M)$ with $A \cap B=(0), A+B=M ; B$ is called a semicomplement of $A$. If each $A \in S L(M)$ is semicomplemented then $S L(M)$ is said to be semicomplemented. An $N$-subgroup $A$ of $M$ is module-essential if whenever $B$ is a non-zero submodule of $M$ then $A \cap B \neq(0)$.

A submodule $A$ of $M$ is minimal if it contains no $N$-subgroups other than (0) and $A$. If $M$ is a direct sum of minimal submodules then $M$ is completely reducible. The near-ring $N$ is completely reducible if it is completely reducible as an $N$-module.

In (7; Theorem 3) we proved

Theorem 1. For an $N$-module $M$ the following are equivalent:-

(i) $M$ is completely reducible;

(ii) $M$ has no proper module-essential $N$-subgroups;

(iii) $S L(M)$ is semicomplemented.

Proposition 1. If $N$ has a left identity the following are equivalent:-

(i) $N$ is completely reducible;

(ii) each maximal $N$-subgroup of $N$ is semicomplemented;

(iii) each proper $N$-subgroup of $N$ is contained in a semicomplemented proper $N$-subgroup of $N$.

Proof. Clearly (i) $\Rightarrow$ (ii) $\Rightarrow$ (iii). Suppose (iii) and let $A$ be a proper 
module-essential $N$-subgroup of $N$. Then $A$ is contained in a proper $N$-subgroup of $N$ which is semicomplemented. This contradiction and Theorem 1 establishes that (iii) $\Rightarrow$ (i).

Completely reducible near-rings with left identity clearly have the minimum condition on $N$-subgroups. Retaining the chain condition but not the left identity we can prove

Proposition 2. If $N$ has the minimum condition on $N$-subgroups the following are equivalent:-

(i) $N$ is completely reducible;

(ii) each non-zero $N$-subgroup of $N$ contains a non-zero semicomplemented $N$-subgroup of $N$.

Proof. That (i) $\Rightarrow$ (ii) is trivial. Suppose (ii) and let $X$ be an $N$-subgroup of $N$ which is not semicomplemented. Let $T \subset X$ be a non-zero $N$-subgroup of $N$ semicomplemented by $A \in L(N)$. Then $X=T+X_{1}$ where $X_{1}=A \cap X$ and $T \cap X_{1}=(0)$. If $X_{1}$ is semicomplemented by $Y \in L(N)$ and if $u \in X \cap(A \cap Y)$ then $u \in\left(T+X_{1}\right) \cap A \cap Y$ so that

$$
u=t+x_{1}=a \quad\left(t \in T, x_{1} \in X_{1}, a \in A \cap Y\right) .
$$

Thus $t=a-x_{1} \in T \cap A=(0) \quad$ and $\quad u=x_{1} \in X_{1} \cap A \cap Y=(0)$. Then $X \cap(A \cap Y)=(0)$. Now let $z \in N=T+A$ so that $z=t+a(t \in T, a \in A)$. Since $N=X_{1}+Y, a=x_{1}+y\left(x_{1} \in X_{1}, y \in Y\right)$ and

$$
z=t+x_{1}+y \in\left(T+X_{1}\right)+(A \cap Y)=X+A \cap Y .
$$

It follows that $X$ is semicomplemented if $X_{1}$ is semicomplemented. If $X_{1}$ is not semicomplemented we can apply the same construction to $X_{1}$ to obtain $X_{2}$ and then $X_{3}$ etc with $\ldots \subset X_{n} \subset \ldots \subset X_{1}$ contrary to the minimum condition for $N$-subgroups. It follows that (ii) $\Rightarrow$ (i).

In (7; Theorem 4) we gave a proof of

Proposition 3. If $N$ is a near-ring with left identity the following are equivalent:-

(i) $N$ is completely reducible;

(ii) $N$ has no nilpotent $N$-subgroups and has the minimum condition on N-subgroups.

Later we will give an alternative proof of this result. A near-ring $N$ is regular if for each $r \in N$ there exists $s \in N$ with $r=r s r$. It is easy to see

Lemma 1. If $N$ is a near-ring with identity the following are equivalent:-

(i) $N$ is regular;

(ii) for each $a \in N$ there is a non-zero idempotent $e \in N$ with $a N=e N$;

(iii) for each $a \in N$ there is a right ideal $B$ of $N$ with $a N \cap B=(0)$ and $a N+B=N$. 
We observe that (i) $\Rightarrow$ (ii) $\Rightarrow$ (iii) irrespective of whether $N$ has an identity. Furthermore if we assume that $N$ has minimum condition on $N$ subgroups we can use Proposition 2 to prove

Corollary 1. If $N$ has minimum condition on $N$-subgroups and if $N$ is regular then $N$ is completely reducible.

Later we will consider the converse of this. For the present we observe

Proposition 4. If $N$ is a near-ring with identity the following are equivalent:-

(i) $N$ is completely reducible;

(ii) $N$ has the minimum condition on $N$-subgroups and is regular.

If $R$ is a ring then $R$ is completely reducible if and only if every $R$-module is completely reducible. We are unable to prove this for near-rings. However, calling an $N$-module $M$ monogenic if $M=m N$ for some $m \in M$ we have

Proposition 5. If $N$ is a near-ring with left identity then $N$ is completely reducible if and only if every monogenic $\mathbf{N}$-module is completely reducible.

Proof. Clearly if every monogenic $N$-module is completely reducible so is $N$. For the converse let $M=m N$ with $m \in M$. For $I \in S L(M), T=$ $\{n \in N: m n \in I\} \in S L(N)$ and $I=m T$. Let $P \in L(N)$ with $T \cap P=(0)$, $T+P=N$. Then $M=m T+m P=I+m P, I \cap m P=(0), m P \in L(M)$.

An $N$-subgroup $A$ of a module $M$ is essential if $A \cap B \neq(0)$ whenever $B$ is a non-zero $N$-subgroup of $M$. Then

Corollary 2. If $N$ has an identity and is completely reducible and if $M$ is an $N$-module then $M$ has no essential $N$-subgroups.

Proof. Let $A \in S L(M)$ be essential and $x \in M$ with $x \neq 0$. From Proposition $5, x N$ is completely reducible. Let $K \in L(x N)$ with $x N \cap A \cap K=$ (0), $x N \cap A+K=x N$. But $x N \cap K=K$ so $A \cap K=(0)$ and $K \in S L(M)$ so $K=(0)$. Thus $x N \cap A=x N$ and $x \in A$. But then $M \subseteq A$.

Let $M$ be a completely reducible $N$-module with $M=\oplus_{\Lambda} M_{\lambda}$ where $M_{\lambda}$ is a minimal submodule of $M$ and $P$ be any minimal $N$-subgroup of $M$. Denote by $\left\{\Pi_{\alpha}: M \rightarrow M_{\alpha}\right\}$ the family of natural projections and by $\theta_{\alpha}$ the restriction of $\Pi_{\alpha}$ to $P$. Clearly $\theta_{\alpha}=0$ or $\theta_{\alpha}$ is an $N$-isomorphism. For each minimal $N$-subgroup $P$ of $M$ let $H(P)$ denote the sum of all those submodules of $M$ which are isomorphic as $N$-modules to $P . H(P)$ is the homogeneous component of $P$ and clearly

Proposition 6. If $M$ is completely reducible then $M=\oplus H(P)$ where $P$ ranges over all the minimal $N$-subgroups of $M$.

We notice that we can define homogeneous components for general modules in just the same way. If $P$ is a minimal $N$-subgroup of $M$ then 
$P \subset H(P)$ if $M$ is completely reducible. However this need not be so if $M$ is not completely reducible; for example the symmetric group $S_{3}$ on 3 elements is a $(Z, 1)$-module (notation in Fröhlich (5)), where $Z$ is the set of integers, in which the subset $P=\{e, \alpha\}$ with $\alpha^{2}=e$ is a minimal $(Z, 1)$ subgroup for which $H(P)=(0)$.

Lemma 2. If $F$ is a homogeneous component in a completely reducible near-ring $N$ then $F$ is an ideal.

Proof. Clearly $\alpha \in \operatorname{Hom}_{N}(N, N)$ implies $\alpha F \subseteq F$ in a completely reducible near-ring. For $x \in N$ define $\alpha_{x} \in \operatorname{Hom}_{N}(N, N)$ by $\alpha_{x}(n)=x n$.

For an $N$-module $M$ we denote by $\operatorname{Soc}(M)$ the sum of all the minimal submodules of $M$. As before it is not necessary for $\operatorname{Soc}(M)$ to contain all the minimal $N$-subgroups of $M$. Trivially $\operatorname{Soc}(M)=M$ if and only if $M$ is completely reducible. If $M$ is not completely reducible denote by $T$ the intersection of all the module essential $N$-subgroups of $M$.

We shall, on several occasions, use

Lemma 3. If $M$ is an $N$-module and $A \in S L(M)$ there exists $B \in L(M)$ with $A \cap B=(0)$ and $A+B$ module-essential in $M$.

Proof. The family of submodules of $M$ having trivial intersection with $A$ is non-empty since it contains (0). For any chain $B_{1} \subseteq B_{2} \subseteq \cdots$ of submodules of $M$ with $A \cap B_{i}=(0)$ for each $i$ we see that $A \cap\left(\cup B_{i}\right)=(0)$. Hence by Zorn's Lemma there is a maximal submodule $B$ of $M$ with $A \cap B=(0)$. Clearly if $X \in L(M)$ with $(A+B) \cap X=(0)$ then

$$
A \cap(B+X)=(0)
$$

and since $B+X \in L(M)$ this contradicts the maximality of $B$.

Proposition 7. T is completely reducible as an $N$-module.

Proof. If $X \in S L(T)$ then $X \in S L(M)$ and by Lemma 3 we can choose $Q \in L(M)$ maximal subject to $X \cap Q=(0)$. Then $X+Q$ is module essential so $T \subseteq X+Q$ and $T=X+T \cap Q$ where $T \cap Q \in L(T)$.

For $P$ a minimal submodule of $M$ we have $P=T \cap P$ so $\operatorname{Soc}(M) \subseteq T$.

Proposition 8. If $T$ is a submodule of $M$ then $\operatorname{Soc}(M)=T$.

Proof. Let $p \in T \backslash \operatorname{Soc}(M)$ and $Q \in L(M)$ be maximal subject to the two conditions $\operatorname{Soc}(M) \subseteq Q$ and $p \notin Q . Q_{1}=Q \cap T \in L(M)$. Using Lemma 3 let $A \in L(M)$ with $Q_{1} \cap A=(0), Q_{1}+A$ module essential in $M$. Then $T=Q_{1}+A_{1}$ where $A_{1}=A \cap T$. If $X \neq(0)$ and $X \in S L\left(A_{1}\right)$ then $X \in S L(M)$ so there exists $B \in L(M)$ with $X \cap B=(0), \quad X+B \cap A_{1}=A_{1} . \quad A_{1}$, $B \in L(M)$ so $B \cap A_{1}=B_{1} \in L(M)$. If $X \neq A_{1}$ then $B_{1} \neq(0)$ so for some $C_{1} \in L(M), B_{1} \cap C_{1}=(0), B_{1}+C_{1}=A_{1}$. Now $X \neq(0)$ implies $B_{1} \neq(0) \neq C_{1}$. Clearly $B_{1} \cap Q=C_{1} \cap Q=(0)$ and $p \in\left(Q+B_{1}\right) \cap\left(Q+C_{1}\right)$. Writing $p=$ $q_{1}+b=q_{2}+c$ then $-q_{2}+q_{1} \in\left(B_{1}+C_{1}\right) \cap Q=A_{1} \cap Q=(0)$ and $b=c=0$ 
contrary to $p \notin Q$. Hence $X=A_{1}$ and $A_{1}$ is a minimal submodule of $M$ from which $A_{1} \subseteq$ Soc $(M) \subseteq Q$ and $A_{1}=(0)$ since $Q \cap A_{1}=(0)$. Then $T=$ $Q_{1}=T \cap Q$ so $T \subset Q$ contrary to $p \notin Q$. It follows that $T=\operatorname{Soc}(M)$.

Whether $T$ is always a submodule of $M$ is unknown.

Proposition 9. If $T$ is not a submodule of $M$ then Soc $(M)$ is the largest submodule of $M$ contained in $T$.

Proof. Let $Q \in L(M)$ with $\operatorname{Soc}(M) \subset Q \subseteq T$ and $q \in Q \backslash \operatorname{Soc}(M)$. If $A \in L(M)$ is maximal subject to the two conditions $q \notin A$ and $\operatorname{Soc}(M) \subseteq A$ then $A_{1}=A \cap Q \in L(M)$. Let $B \in L(M)$ with $A_{1} \cap B=(0), A_{1}+B$ module essential in $M$. Then $B_{1}=B \cap Q \in L(M)$. As in the proof of Proposition 8 we can show that $B_{1}$ is minimal leading to $B_{1}=(0)$ and $Q=\operatorname{Soc}(M)$.

In Proposition 3 we have seen that if $N$ has a left identity then the property of being completely reducible is equivalent to having minimum condition on $N$-subgroups and no nilpotent $N$-subgroups. To drop the requirements of a left identity and minimum condition we recall some results on radicals for near-rings.

If $\Gamma$ is a near-ring module then $\Gamma$ is

type 2: if $\Gamma$ has no proper $N$-subgroups and $\Gamma N \neq(0)$;

type 1: if $\Gamma$ has no proper submodules, $\Gamma N \neq(0)$ and $\gamma \in \Gamma$ implies $\gamma N=$ (0) or $\gamma N=\Gamma$.

type 0: if $\Gamma$ has no proper submodules and $\gamma N=\Gamma$ for some $\gamma \in \Gamma$.

We define

$$
J_{i}(N)=\bigcap\left\{r_{N}(\Gamma): \Gamma \text { is a type } i N \text {-module }\right\}
$$

where $r_{N}(\Gamma)=\left\{n \in N: \Gamma_{n}=(0)\right\}$. If $\Gamma$ has no type $i N$-modules we put $J_{i}(N)=N$. A right ideal $I$ of $N$ is modular if there exists $a \in N$ with $x$-ax $\in I$ whenever $x \in N . D(N)$ is the intersection of all the modular maximal right ideals of $N$ with $D(N)=N$ if $N$ has no modular maximal right ideals. It is known that $J_{0}(N) \subseteq D(N) \subseteq J_{1}(N) \subseteq J_{2}(N)$. Furthermore $J_{0}(N)$ contains all the nilpotent ideals of $N, J_{2}(N)$ all the nilpotent $N$-subgroups.

If $A$ is a minimal non-nilpotent $N$-subgroup of $N$ then $A=e N$ for some idempotent $e \in A$. Let $A \in S L(N)$ be non-nilpotent and $A \subseteq J_{2}(N)$. $N=e N+r(e)$ and $n-e n \in r(e)$ for each $n \in N$. Thus $r(e)$ is modular and $N / r(e) \cong e N$. Since $e N$ is type 2 we say that $r(e)$ is 2 -primitive. Betsch (1; Satz 3.2) proved that $J_{2}(N)$ is the intersection of the 2-primitive right ideals of $N$ so $J_{2}(N) \subseteq r(e)$ contrary to $e^{2} N \neq(0)$.

Theorem 2. If $N$ is completely reducible then $J_{2}(N)$ is the sum of all the nilpotent right ideals of $N$ and $J_{2}(N)^{2}=(0)$.

Proof. $J_{2}(N)$ is the sum of all the minimal right ideals of $N$ which it contains and we have seen that each of these is nilpotent. Clearly if $A$ is a nilpotent minimal right ideal then $A^{2}=(0)$. Let $A_{1}, A_{2}$ be nilpotent minimal 
right ideals of $N$. If $A_{1} \not A_{2}$ then $H\left(A_{1}\right) \cap H\left(A_{2}\right)=(0)$ and so $H\left(A_{1}\right) H\left(A_{2}\right)=(0)$ and $A_{1} A_{2}=(0)$. If $A_{1} \cong A_{2}$ let $\phi$ be the isomorphism and $a_{1} \in A_{1}, a_{2} \in A_{2}, a_{2}^{*} \in A_{2}$ with $\phi\left(a_{2}^{*}\right)=a_{1}$. Then $a_{1} a_{2}=\phi\left(a_{2}^{*}\right) a_{2}=\phi\left(a_{2}^{*} a_{2}\right)=$ $\phi(0)=0$. It follows that $A_{1} A_{2}=(0)$ and $J_{2}(N)^{2}=(0)$. $J_{2}(N)$.

Corollary 3. If $N$ is completely reducible then $J_{0}(N)=D(N)=J_{1}(N)=$

Proof. $J_{2}(N)$ is a nilpotent ideal so $J_{2}(N) \subseteq J_{0}(N)$.

As a second corollary to this we will obtain a proof of Proposition 3 different from that in (7). An element $x \in N$ is right quasi-regular (rqr) if and only if the minimal right ideal of $N$ containing all elements of the form $n-x n$ for each $n \in N$ also contains $x$. If we denote by $L_{x}$ the right ideal of $N$ generated by $\{n-x n: n \in N\}$ then $x$ is rqr if and only if $x \in L_{x}$.

Lemma 4. $x$ is rqr if and only if $L_{x}=N$.

Proof. If $L_{x}=N$ then $x \in L_{x}$. Conversely if $x$ is rqr then $x \in L_{x}$ so for $s \in N, s=(s-x s)+x s \in L_{x}$ and $N=L_{x}$.

A right ideal of $N$ is quasi-regular in case each of its elements is rqr. By Ramakotoiah $(8 ; 2.2) D(N)$ is quasi-regular and contains all the quasi-regular right ideals of $N$. A right ideal, $A$, of $N$ is small if and only if whenever $B \in L(N)$ with $A+B=N$ then $B=N$.

Lemma 5. If $I$ is a right ideal of $N$ and $N$ has a left identity $e$ then $I$ is small if and only if $I \subseteq D(N)$.

Proof. Let $I \subseteq D(N)$ and $B \in L(N)$ with $B+I=N$. Then $e=b+i$. Now $D(N)$ is quasi-regular, so $i$ is rqr; so by Lemma $4, L_{i}=N$. But $r \in N$ implies $r-i r=(b+i) r-i r \in B$. Thus $L_{i} \subseteq B$ and $B=N$ as required. Conversely, if $I$ is a small right ideal let $x \in I$. Then $e-x e \in L_{x}$ so $e=(e-x e)+x e \in L_{x}+I$. Hence $L_{x}=N$ and $I \subseteq D(N)$.

This gives an alternative characterisation of $D(N)$.

Corollary 4. If $N$ has a left identity then $D(N)$ is a small right ideal of $N$ and is the sum of all the small right ideals of $N$.

Corollary 5. For a near-ring $N$ with left identity the following are equivalent

(i) $N$ is completely reducible;

(ii) $N$ has no nilpotent $N$-subgroups and satisfies the minimum conon $\mathrm{N}$-subgroups.

Proof. (ii) implies (i) is due to Blackett (3). Suppose (i). Then $D(N)=$ (0) so that $J_{2}(N)=(0)$ and $N$ has no nilpotent $N$-subgroups. The minimum condition follows immediately from $N$ having a left identity.

Let us now turn to the case where $J_{2}(N)=(0)$. 
Theorem 3. If $N$ is completely reducible and $J_{2}(N)=(0)$ then each homogeneous component is a simple near-ring.

Proof. Let $N=\oplus F_{\lambda}$ where each $F_{\lambda}$ is a homogeneous component. For distinct $F_{1}, F_{2}$ we have $F_{1} F_{2}=(0)$ so if $X$ is an ideal of $F_{1}$ then $X$ is a right ideal (in fact an ideal) of $N$. If $X \neq F_{1}$ let $A$ be a minimal right ideal of $N$ in $F_{1}$ with $X \cap A=(0)$. Since $J_{2}(N)=(0), A=e N$ for some non-zero idempotent $e \in A$. If $X \neq(0)$ let $f N$ be a minimal right ideal of $N$ in $X$ with $f=f^{2} \neq 0$. Then $A X \subseteq A \cap X=(0)$ so $X \subseteq r(A)$. The minimal right ideals of $N$ in $F_{1}$ are isomorphic and thus $f N \cong e N$. If $\phi$ is the isomorphism let $\phi(f)=e n$. Then $0=e n f=\phi(f) f=\phi(f)$ which is not true. Thus $X=F_{1}$ or $X=(0)$ and $F_{1}$ is a simple near-ring.

Corollary 6. If $N$ is completely reducible with $J_{2}(N)=(0)$ and if $A$ is a two-sided $N$-subgroup of $N$ there is a two-sided ideal $X$ of $N$ with $A \cap X=(0)$ and $A+X=N$.

Proof. Let $X$ be an ideal of $N$ maximal subject to $A \cap X=(0)$. Write $N=\oplus F_{\lambda}$ where each $F_{\lambda}$ is a homogeneous component and thus an ideal of $N$ and simple as a near-ring. Clearly $(A+X) \cap F_{\lambda} \neq(0)$ for each $\lambda$. If $B$ is a minimal $N$-subgroup of $N$ contained in $F_{\lambda}$ and $B(A+X)=(0)$ then $(A+$ $X) \cap F_{\lambda} \subseteq r(B) \cap F_{\lambda}$. Now $F_{\lambda}$ is simple and thus has no proper two-sided ideals so $r(B) \cap F_{\lambda}=(0)$ or $F_{\lambda}$. Since $(A+X) \cap F_{\lambda} \neq(0)$ we must have $r(B) \cap F_{\lambda}=F_{\lambda}$ and so $B \subseteq r(B) \cap F_{\lambda}$. But then $B^{2}=(0)$ contrary to $J_{2}(N)=$ (0). Since $(A+X) \cap B=(0)$ implies $B(A+X)=(0)$ it follows that $B \subseteq A+$ $X$ for each minimal right ideal of $N$ and thus $A+X=N$ as required.

Theorem 4. If $J_{2}(N)=(0)$ and $N=\oplus N_{\lambda}$, where each $N_{\lambda}$ is an ideal of $N$, is simple as a near-ring and contains a minimal right ideal then $N$ is completely reducible.

Proof. If $A$ is the minimal right ideal of $N_{\lambda}$ and $B$ is isomorphic to $A$ as an $N$-module then $B \subseteq N_{\lambda}$ since $J_{2}(N)=(0)$. Apply Zorn's Lemma to the family of all sums of right ideals of $N_{\lambda}$ which are isomorphic to $A$ to obtain a maximal such sum $T$. Then $T$ is an ideal of $N_{\lambda}$ so $T=N_{\lambda}$ and $N$ is completely reducible.

We now obtain the structure of two-sided $N$-subgroups of a completely reducible near-ring with identity.

Lemma 6. If $N$ has no nilpotent $N$-subgroups and $A$ is an $N$-subgroup of $N, B$ a two-sided $N$-subgroup of $N$, then $A B=(0)$ if and only if $A \cap B=(0)$.

Proposition 10. If $N$ is completely reducible with identity 1 and $A$ is a two-sided $N$-subgroup of $N$ then $A=e N$ where $e$ is a central idempotent.

Proof. From Theorem 2 and Corollary 5 we get $J_{2}(N)=(0)$. From 20/3-B 
Corollary 6 there is an ideal $X$ of $N$ with $A \cap X=(0), A+X=N$. Write $1=e+x(e \in A, x \in X)$. Then $e-e^{2}=(e+x) e-e^{2} \in A \cap X=(0)$. Clearly $A=e N$ and $e$ is central.

So far we have not distinguished between rings and near-rings. We now wish to investigate near-rings which are not rings. These we call nonrings. An extremely important result (due to Wielandt and reported by Betsch (2; 2.12)) is

Lemma 7. Let $N$ be a near-ring and $\Gamma$ a faithful $N$-module with $\Gamma=\gamma N$ for some $\gamma \in \Gamma$. If $B, C \in L(N)$ satisfy

$$
B+r_{N}(\gamma)=N=C+r_{N}(\gamma) ; \quad B \cap C \subseteq r_{N}(\gamma)
$$

then $N$ is a ring.

Lemma 8. Let $\Gamma$ be a type $2 N$-module and $\gamma \in \Gamma$ with $\gamma N \neq(0)$. If $I \in S L(N)$ with $r_{N}(\gamma) \subsetneq$ I then $I=N$.

Proof. Since $r_{N}(\gamma) \underset{\neq I}{\subset}$ we have $\gamma I=\Gamma$. If $n \in N$, then for some $t \in I$, $\gamma n=\gamma t$ so $n-t \in r_{N}(\gamma) \subset I$ and thus $n \in I$ and $N=I$.

By a standard argument one can show that if $A$ is a non-nilpotent minimal $N$-subgroup of a near-ring $N$ then $A=e N$ for some idempotent $e \in A$.

Lemma 9. If $N$ is a completely reducible nonring, without proper 2-sided ideals, with $J_{2}(N)=(0)$ and if $e N$ is a minimal right ideal of $N$ and $X$ a right ideal of $N$ with $e N \cap X=(0)$ then $X \subseteq r(e)$.

Proof. If $x \in X$ with $e x \neq 0$ then $r(e)+X=N=r(e)+e N$, and $e N \cap X=(0) \subseteq r(e)$ contrary to $N$ being a nonring.

Theorem 5. If $N$ is a completely reducible nonring, without proper 2-sided ideals, with $J_{2}(N)=(0)$ then the lattice of right ideals of $N$ has unique complements.

Proof. Let $X \in L(N)$ with $A, B \in L(N)$ such that $X \cap A=(0)=$ $X \cap B, X+A=N=X+B$. If $e N$ is a minimal right ideal of $N$ with $e N \cap A=(0)$ then $A \subseteq r(e)$. Since $r(e) \neq N$ we cannot have $X \subseteq r(e)$ and so $X \cap e N \neq(0)$ and $e N \subseteq X$. It follows that $A$ is the sum of all those minimal right ideals of $N$ not in $X$. Similarly $B$ is also their sum and $A=B$.

Corollary 7. If $N$ is a completely reducible nonring, without proper 2-sided ideals, with $J_{2}(N)=(0)$ then the lattice $L(N)$ is distributive.

The proof of Theorem 5 contains the proofs of the following

Lemma 10. If $N$ is a completely reducible nonring, without proper 2-sided ideals, with $J_{2}(N)=(0)$ and $A \in L(N)$ then $A$ is the sum of the minimal right ideals of $N$ which are contained in it. 
Lemma 11. If $N$ is a completely reducible nonring without proper 2-sided ideals and $N=\oplus A_{\lambda}$ where each $A_{\lambda}$ is a minimal non-nilpotent right ideal of $N$ then each minimal right ideal of $N$ is one of these $A_{\lambda}$.

A near-ring $N$ is $v$-primitive $(v=0,1,2)$ if it has a faithful type $v$ $N$-module. A simple nonring without nilpotent $N$-subgroups and with a minimal $N$-subgroup will be 2-primitive and hence 1-primitive. For 1primitive nonrings Ramakotaiah proved a density theorem which we wish to use.

Let $N$ be a 1-primitive nonring and $\Gamma$ be a faithful type $1 N$-module. If $x, y \in N$ we define $x \sim y$ if and only if $r_{N}(x)=r_{N}(y)$. Clearly $\sim$ is an equivalence relation and $C_{0}$, the equivalence class containing 0 , consists precisely of those $x \in \Gamma$ with $x N=(0)$. Ramakotaiah (9; Theorem 4) proved

Lemma 12. Let $N$ be a 1-primitive nonring and $\Gamma$ be a faithful type 1 $N$-module. Let $w_{1}, w_{2}, \ldots, w_{n} \in \Gamma \backslash C_{0}$ with $w_{i} \propto w_{j}$ if $i \neq j$. For each set $m_{1}, m_{2}, \ldots, m_{n} \in \Gamma$ there is an element $b \in N$ with $w_{i} b=m_{i}(1 \leqslant i \leqslant n)$.

Lemma 13. Let $N$ be a completely reducible nonring, without proper two-sided ideals, in which $J_{2}(N)=(0)$. Then $N$ has a system of idempotents $\left\{e_{\lambda}\right\}$ such that $e_{\lambda} e_{\mu}=0$ if $\lambda \neq \mu$.

Proof. Writing $N=\oplus_{\lambda} e_{\lambda} N$ where each $e_{\lambda} N$ is a minimal right ideal of $N$ and $e_{\lambda}^{2}=e_{\lambda}$ we know that $e_{\lambda} N \cap r_{N}\left(e_{\lambda}\right)=(0)$ and $e_{\lambda} N \oplus r_{N}\left(e_{\lambda}\right)=N$. If $\lambda \neq \mu$ then $e_{\mu} N \cap e_{\lambda} N=(0)$ and so, from Lemma 9, $e_{\mu} N \subseteq r_{N}\left(e_{\lambda}\right)$ and $e_{\lambda} e_{\mu}=0$ as required.

Now suppose that $N$ is a completely reducible nonring with $J_{2}(N)=(0)$ in which $x t=y t$ for each $t \in N$ implies $x=y$. Writing $N=\oplus N_{\lambda}$ where each $N_{\lambda}$ is a homogeneous component of $N$ we see that each $N_{\lambda}$ has these properties and in addition has no two-sided proper ideals. Those $N_{\lambda}$ which are simple rings are regular by Blair (4). Thus we need only consider those $N_{\lambda}$ which are completely reducible nonrings with $J_{2}\left(N_{\lambda}\right)=(0)$, which have no two-sided proper ideals and in which $x, y \in N$ with $x t=y t$ for each $t \in N$ implies $x=y$.

Theorem 6. If $N$ is a completely reducible nonring, without proper two-sided ideals, such that $J_{2}(N)=(0)$ and whenever $x, y \in N$ with $x t=y t$ for each $t \in N$ then $z=y$ then $N$ is regular in the sense that to each $a \in N$ there corresponds $b \in N$ with $a=a b a$.

Proof. Let $a \in N$. Choose non-nilpotent minimal right ideals $e_{1} N, \ldots, e_{k} N$ with $a \in e_{1} N \oplus \ldots \oplus e_{k} N$, and $k$ minimal, where $e_{i}^{2}=e_{i}$ for each $i$. Then as $N$-modules, $e_{i} N$ is isomorphic to $e_{j} N$ for $1 \leqslant i, j \leqslant k$. Let $\phi_{j}$ : $e_{j} N \rightarrow e_{1} N$ be an isomorphism and write $\gamma_{j}=\phi_{j}\left(e_{j}\right)$. Clearly $\gamma_{i} \sim \gamma_{j}$ if and only if $i=j$. From Lemma 13 we observe that if $N=\bigoplus e_{\lambda} N$ where each $e_{\lambda} N$ is a non-nilpotent minimal right ideal of $N$ then $e_{\lambda} a=0$ if 
$\lambda \neq 1,2, \ldots, k$ and, since $k$ is minimal, $e_{j} a \neq 0$ for $1 \leqslant i \leqslant k$. Hence $\gamma_{i} a \neq 0$ for $1 \leqslant i \leqslant k$. Let $\gamma_{1} a, \gamma_{2} a, \ldots, \gamma_{q} a$ be those $\gamma_{i} a$ in different equivalence classes under $\sim$. Clearly $e_{1} N x=0$ implies $x=0$ so $N$ is a 2-primitive near-ring and thus 1-primitive. Appealing to Lemma 12 we can choose $b \in N$ with $\gamma_{i} a b=\gamma_{i}$ for $1 \leqslant i \leqslant q$. Now consider $\gamma_{j} a$ where $q<j \leqslant k$. For some $i$, $\gamma_{j} a \sim \gamma_{i} a$, so $r\left(\gamma_{j} a\right)=r\left(\gamma_{i} a\right)$. Now $\gamma_{i} a b a t=\gamma_{i} a t$ for each $t \in N$; so $b a t-t \in$ $r\left(\gamma_{i} a\right)$. It follows that $b a t-t \in r\left(\gamma_{j} a\right)$ for each $t \in N$. Hence $1 \leqslant s \leqslant k$ and $t \in N$ implies $\gamma_{s} a b a t=\gamma_{s} a t$. By assumption we have $\gamma_{s} a b a=\gamma_{s} a$. Hence $a b a-a \in r\left(\gamma_{s}\right)=r\left(e_{s}\right)$; so

$$
a b a-a \in e_{1} N \oplus \cdots \oplus e_{k} N \cap r\left(e_{1}\right) \cap \cdots \cap r\left(e_{k}\right)=(0),
$$

or $a b a=a$ as required.

Corollary 8. If $N$ is a completely reducible nonring with $J_{2}(N)=(0)$ and if $x, y \in N$ with $x t=y t$ for each $t \in N$ implies $x=y$ then $N$ is regular.

Proof. A direct sum of regular near-rings each of which is an ideal in the sum is regular so we simply apply Blair's result to those direct summands which are rings and Theorem 6 to the nonrings.

Observe that if $R$ is a ring with $J_{2}(R)=(0)$ then $x R=(0)$ if and only if $x=0$. Whether this is true for a general near-ring is unknown. However, when $N$ is distributively generated we have

Lemma 14. If $N$ is distributively generated and has no nilpotent $N$ subgroups then $x N x=(0)$ implies $x=0$.

Proof. Let $N$ be distributively generated by $S$ (i.e. $a, b \in N, s \in S$ implies $(a+b) s=a s+b s$ and $a \in N$ implies $a=\sigma_{1}+\sigma_{2}+\cdots+\sigma_{n}$ where for $1 \leqslant i \leqslant n$ either $\sigma_{i} \in S$ or $\left.-\sigma_{i} \in S\right)$. From $x N x=(0)$ we get $(x N)^{2}=(0)$ and hence $x N=(0)$. Let $B$ be the $N$-subgroup of $N$ generated by $x$. If $b \in B$ then $b=n \cdot x, n$ an integer, in the obvious notation, since $x N=0$. Then $(n \cdot x)(m \cdot x)=m .((n . x) \cdot x)$. Now $x=\Sigma_{j} \sigma_{j}$, where either $\sigma_{j} \in S$ or $-\sigma_{j} \in S$. Then $(n . x)\left(\Sigma_{j} \sigma_{j}\right)=\Sigma \pm\left(n\left( \pm x \sigma_{j}\right)\right)$, taking the positive signs when $\sigma_{j} \in S$ and the negative signs when $-\sigma_{j} \in S$, but $\sigma_{j} \notin S$. As $x \sigma_{j} \in x N=0$, so $(n \cdot x) x=0$ and $(n \cdot x)(m \cdot x)=0$. Thus $B^{2}=(0)$ and so $B=(0)$.

Corollary 9. If $N$ is distributively generated by $S$ and has no nilpotent $N$-subgroups then $x, y \in N$ with $x t=y t$ for each $t \in N$ implies $x=y$.

Proof. In particular $x s=y s$ for $s \in S$ and so $(x-y) s=0$. It follows that $S \subseteq r(x-y)$ and hence $N \subseteq r(x-y)$. Then $(x-y) N=(0)$ so $x-y=0$ and $x=y$.

Combining this with Corollary 8 we obtain

Theorem 7. If $N$ is a distributively generated, completely reducible near-ring and $J_{2}(N)=(0)$ then $N$ is regular. 
I should like to take this opportunity of thanking the referee for his many helpful comments on an earlier version of this paper.

\section{REFERENCES}

(1) G. BeTSCH, Ein Radikal für Fastringe, Math. Z. 78 (1962), 86-90.

(2) G. BETSCH, Primitive Near-rings, Math. Z. 130 (1973), 351-361.

(3) D. W. Blacketr, Simple and Semi-simple Near-rings, Proc. Amer. Math. Soc. 4 (1953), 772-785.

(4) R. L. BLAIR, Ideal Lattices and the Structure of Rings, Trans. Amer. Math. Soc. 75 (1953), 136-153.

(5) A. FRÖHLICH, Distributively Generated Near-rings (I Ideal Theory), Proc. London Math. Soc. (3) 8 (1958), 76-94.

(6) A. Oswald, Near-rings in which every $\mathrm{N}$-subgroup is Principal, Proc. London Math. Soc. (3) 28 (1974), 67-88.

(7) A. Oswald, Semisimple Near-rings have the Maximum Condition on $N$-subgroups, J. London Math. Soc. 11 (1975), 408-412.

(8) D. Ramakotaiah, Radicals for Near-rings, Math. Z. 97 (1967), 45-56.

(9) D. RamaKotalah, Structure of 1-primitive Near-rings, Math. Z. 110 (1969), $15-26$.

DePaRTMENT OF MATHEMATICS

Teesside Polytechnic

MidDlesbrough

Cleveland

TS1 3BA 